\title{
Origin of Radio Emission from Nonthermal Electrons in ADAF for LLAGNs
}

\author{
H. Liu and Q. W. Wu* \\ School of Physics, Huazhong University of Science and Technology, Wuhan 430074, China \\ *email: qwwu@hust.edu.cn
}

\begin{abstract}
We explore the origin of the radio emission from the low-luminosity active galactic nuclei (LLAGNs), by investigating the ADAF model with a hybrid of thermal and nonthermal electrons. We find that the radio emission of LLAGNs is severely underpredicted by pure ADAF model, and can be naturally explained by the ADAF model with a hybrid electron population. Our model can roughly reproduce the anti-correlation between the mass-corrected radio loudness and the Eddington ratio for the LLAGNs. We further model the SEDs of each source, and find that roughly all sources can be well fitted if a small fraction of the steady state electron energy is ejected into the nonthermal electrons.
\end{abstract}

\section{Introduction}

The radio emission of $\mathrm{RL}$ AGNs is believed to originate in the relativistic jet, while the origin of nuclear radio emission from RQ objects is still not well understood, because most of the RQ AGNs only show a weaker compact radio core. The low-luminosity active galactic nuclei (LLAGNs) are very common in the local universe, which may be the scaled-down version of bright AGNs. The advection dominated accretion flow (ADAF) can successfully explain most observational features of nearby LLAGNs (e.g., see Ho 2008 for a recent review). It is natural that both the thermal and nonthermal electrons exist in the hot plasma (ADAF or disk corona), since that the turbulence, magnetic reconnection, and weak shocks can accelerate electrons and generate a nonthermal tail at high energies in the distribution function of thermal electrons. The Galactic center source-Sgr A* provide an excellent observational evidence for the existence of the hybrid of thermal and nonthermal electrons in the ADAF, since that its two components of the radio emission can be well explained by the thermal and nonthermal electrons in ADAF respectively (Yuan et al. 2003). In this work, we test whether some nonthermal electrons in ADAF can also explain the radio emission from the other nearby LLAGNs or not.

\section{Model}

We consider the ADAF model proposed by Yuan et al. (2003), where both outflows $\left[\dot{m}=\dot{m}_{\text {out }}\left(R / R_{\text {out }}\right)^{s}\right]$ and the possible existence of nonthermal electrons are considered. The global structure of an ADAF can be calculated with proper outer boundaries, if the parameters $\dot{m}, \alpha, \beta$, and $\delta$ are specified, where we fix wind parameter $s=0.3$, viscosity parameter $\alpha=0.3$, ratio of gas pressure to total pressure $\beta=0.9$, and the fraction of the viscous energy heats electrons $\delta=0.1$ in this work. To describe the nonthermal electrons in ADAF, we fix the index of power-law distribution for nonthermal electrons $p=3.0$, and allow the parameter $\eta$ to be free which describe the ratio of thermal electron energy to nonthermal electrons. 


\section{Results}

We selected a sample of LLAGNs which have only compact radio cores, where the sources have the possible jets have been excluded (e.g., FR Is). We find that radio emission of our model is roughly proportional to $M_{\mathrm{BH}}^{1.4}$ while that X-ray emission is almost proportional to the mass. To explore the radio emission for a sample of LLAGNs with different $\mathrm{BH}$ masses as a whole, we define a mass-corrected radio-loudness as $R_{\mathrm{M}}=L_{5 \mathrm{GHz}} / L_{2-10 \mathrm{keV}} / M_{\mathrm{BH}}^{0.4}$ and present the relation between $R_{\mathrm{M}}$, and the Eddington ratio, $L_{2-10 \mathrm{keV}} / L_{\mathrm{Edd}}$ in Figure 1 . We find that there is an anti-correlation between $R_{\mathrm{M}}$ and $L_{2-10 \mathrm{keV}} / L_{\mathrm{Edd}}$ for LLAGNs. We also present the theoretical correlations based on our ADAF model with both thermal and nonthermal electrons (see Figure 1), which is roughly consistent with that observations if a small fraction of electron energy ejected into the nonthermal electrons. However, pure ADAF without power-law electrons $(\eta=0$, see Figure 1) always underpredicts the radio emission by several orders of magnitude compared the observations. We further model the spectral energy distributions of 20 LLAGNs

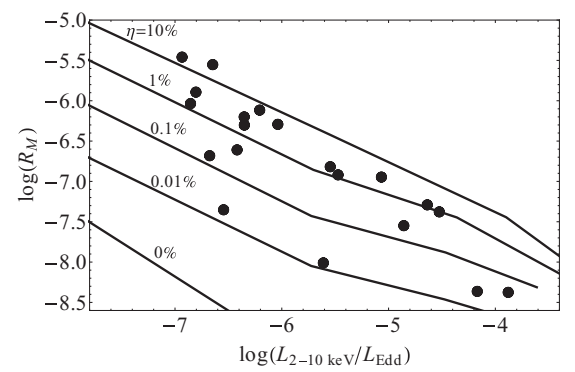

Figure 1. The mass-corrected radio loudness $R_{\mathrm{M}}$ versus the Eddington ratio $L_{2-10 \mathrm{keV}} / L_{\mathrm{Edd}}$. The points represent LLAGNs, and solid lines represent the model predictions.

in our sample, and find that roughly all sources can be well fitted if a small fraction of the steady state electron energy is ejected into the nonthermal electrons. The size of radio emission region of our model is around several thousand gravitational radii, which is also roughly consistent with the recent high-resolution VLBI observations for some nearby LLAGNs. As an example, the spectral fitting for NGC 4594 is shown in Figure 2. In conclusion, we provide a possibility that the compact radio emission of LLAGNs may come from the radiation of the nonthermal electrons in hot accretion flows.

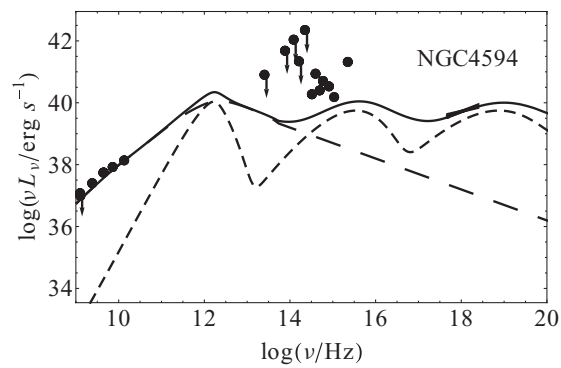

Figure 2. Models for the SED of NGC 4594. The short-dashed and long-dashed lines represent the emission from the thermal and nonthermal electrons of the ADAF respectively.

\section{References}

Ho, L. C. 2008, $A R A \& A, 46,475$

Yuan, F., Quataert, E., \& Narayan, R. 2003, ApJ, 598, 301 